\title{
Identificação de Procedências e Progênies de Pinus maximinoi com potencial produtivo para madeira
}

\author{
Proceedings for the identification of provenances and \\ progenies with wood productive potential in Pinus maximinoi
}

\author{
Wanderley dos Santos ${ }^{1}$, Mayte Sampaio Cesário da Silva², Larissa Delazari Deniz ${ }^{3}$, \\ Wesllen Schuhli Kieras ${ }^{3}$, Jarbas Yukio Shimizu, \\ Valderês Aparecida de Sousa ${ }^{4}$ e Ananda Virginia de Aguiar ${ }^{4}$
}

\begin{abstract}
Resumo
O objetivo desse trabalho foi estimar variabilidade genética de dois ensaios de $P$. maximonoi para identificação de matrizes com potencial produtivo para madeira na região de Ventania-PR. O delineamento experimento foi de blocos completos ao acaso, com 63 progênies área 1 e 77 progênies na área 2 procedentes de oito procedências, nove repetições, seis plantas por parcela, espaçamento de $3 \times 3$ metros. Os caracteres avaliados foram: diâmetro a $1,30 \mathrm{~m}$ do solo (DAP, cm), altura total (ALT, m) e volume (VOL, $\mathrm{m}^{3}$.tree- $\left.{ }^{1}\right)$. As estimativas dos parâmetros genéticos e o ganho na seleção foram estimados com base no método REML/BLUP. Diferenças significativas $(p<0,01)$ foram observadas entre procedências nos dois locais. Houve diferenças significativas entre progênies para os caracteres ALT, DAP e VOL somente para as procedentes de Dulce N. de Colpan (Honduras) e San Jerônimo de Oaxaca (Guatemala) na área 1 e Tapiquil (Honduras) e Valle de Ageles (Honduras) (DAP e VOL) na área 2. Na análise entre procedências os baixos coeficientes de variação genética, herdabilidade aditivos e herdabilidade média de progênies mostram baixo controle genético para os caracteres. As procedências de $P$. maximinoi apresentaram bom crescimento e desempenho em volume na região de Ventania-PR. As procedências de P. maximinoi devem ser consideradas para plantios comerciais e o estabelecimento de áreas de produção de sementes para essa espécie. Dentre as procedências testadas, San Jeronimo (México) teve as maiores taxas de incremento em volume.
\end{abstract}

Palavras-chave: espécies florestais, melhoramento genético, variação genética.

\begin{abstract}
The aim of this work was to estimate the genetic variability in two tests $P$. maximonoi, in order to identify trees with productive potential for wood in the municipality Ventania, state of Paraná, Brazil. A randomized complete block design was used in both tests. Two tests were composed of 63 progenies (area 1) and 77 progenies (area 2) from eight provenances, nine blocks and six plant plots, spaced $3 \times 3 \mathrm{~m}$. The evaluated traits were: Diameter at breast height $(\mathrm{DBH}, \mathrm{cm})$, total height $(\mathrm{H}, \mathrm{m})$ and volume $\left(\mathrm{VOL}, \mathrm{m}^{3} \cdot \mathrm{tree}^{-1}\right)$. Estimates of genetic parameters and the genetic gain in selection were estimated using the REML/BLUP method. Significant differences $(p<0.01)$ were observed between provenances in two areas. There were significant differences between progenies for traits $\mathrm{H}, \mathrm{DBH}$ and VOL only for Dulce N. Colpan (Honduras) and San Jeronimo de Oaxaca (Guatemala) in area 1 and Tapiquil (Honduras) and Valle de Ageles (Honduras) (Dbh and VOL) in area 2. In the analysis between provenances, the low coefficients of genetic variation, additive heritability and medium progenies heritability, show a low genetic control for these traits. In average, $P$. maximinoi provenances showed good growth and volume performance in the Ventania-PR site. The provenances of $P$. maximinoi should be considered not only for commercial plantation but also for the establishment of seed production areas. Among the studied provenances, San Jeronimo (México) had the highest volumes.
\end{abstract}

Keywords: forest species, genetic variation, improvement genetic, provenances.

\footnotetext{
${ }^{1}$ Doutorando em Agronomia. UNESP - Universidade Estadual Paulista "Júlio Mesquita Filho" / Faculdade de Engenharia de Ilha Solteira. Av. Brasil Centro, 56 - Caixa Postal: 31, 15.385-000 - Ilha Solteira, SP, Brasil. E-mail: wanderley.dossantos@hotmail.com.

${ }^{2}$ Mestranda em Engenharia Florestal. UFPR - Universidade Federal do Paraná / Setor de Ciências Agrárias. Av. Lothário Meissner, 3400 - 80210170 - Curitiba, PR, Brasil. E-mail: mahgtm@hotmail.com.

${ }^{3}$ Graduando(a) em Engenharia Florestal. UFPR - Universidade Federal do Paraná / Setor de Ciências Agrárias. Av. Lothário Meissner, 3400 - 80210170 - Curitiba, PR, Brasil. E-mail: lari.delazari@gmail.com; wesllenkieras@hotmail.com.

${ }^{4}$ Pesquisador(a) Doutor. Embrapa Florestas - Empresa Brasileira de Pesquisa Agropecuária. Estrada da Ribeira, Km 111 83411-000 - Colombo, PR, Brasil. E-mail: jyshimizu@gmail.com; valderes.sousa@embrapa.br; ananda.aguiar@embrapa.br
} 


\section{INTRODUÇÃO}

Diversas espécies florestais introduzidas se mostraram adequadas e foram básicas para a formação de florestas de produção no Brasil. Dentre essas encontram as do gênero Pinus, principalmente as de clima subtropical (KAGEYAMA et al., 1977). O gênero Pinus tem uma ampla distribuição geográfica natural, e várias espécies estão exploradas comercialmente em vários países, especialmente no Brasil, Colômbia, Austrália, África do Sul, México, entre outros (SEBBENN et al. 2008).

No Brasil, as plantações florestais ocupam cerca de 7,74 milhões de hectares, dos quais 1,59 milhões correspondem a espécies do gênero Pinus (IBÁ, 2015). A madeira desse gênero é usada como fonte de matéria-prima para as indústrias de chapas, madeira serrada e celulose, dentre outros. Entretanto, para que essa atividade possa continuar suprindo o mercado madeireiro e, simultaneamente elevar o padrão de qualidade da matéria-prima florestal, sem a necessidade de exploração adicional da floresta nativa, é imprescindível o uso de materiais genéticos mais produtivos dentre aqueles com maior potencial de adaptação para reflorestamento no Brasil (CESAR et al.,1988). Altas taxas de ganho genético têm sido obtidas para o caráter volume em Pinus, e também árvores com troncos mais retos, menor número de bifurcações e menor número de galhos grossos têm sido obtidas, fato que conduz a um melhor aproveitamento industrial da madeira (PIRES et al., 2013). Diversas espécies do gênero Pinus estão sendo utilizados no setor madeireiro brasileiro, das quais, o P. taeda e P. elliotii, bem como P. caribaea var. hondurensis se destacam para a produção de madeira e resina. Com o objetivo de ofertar genótipos do gênero Pinus para o mercado visando a exploração de madeira e resina, a avaliação e seleção de materiais mais produtivos tornam-se necessárias. Dentro desse contexto, as espécies do gênero pínus, tanto as usadas em plantios comerciais quanto as que têm potencial produtivo, como $P$. maximinoi, deverão ser contempladas.

P. maximinoi H. E. Moore é a segunda espécie mais comum na América Central, é uma espécie pioneira, que coloniza áreas abertas de florestas (DVORAK, et al. 2000). Segundo Agudelo (1990) Pinus maximinoi é adaptado ao clima subtropical onde não há ocorrência de geadas e a temperatura seja mais amena. Sua madeira apresenta alto potencial de uso em indústrias de celulose, chapas, painéis de fibras e de partículas, palitos e fósforos. A espécie tem grande potencial em relação a qualidade da madeira, tem boa forma de fuste, servindo, dessa forma, como matéria prima para indústria de madeira serrada (MALAN, 2006). É utilizada para construções, celulose de fibra longa e painéis (WRIGHT; WESSELS, 1992), celulose, papel e painéis de colagem lateral (PRATA, 2010). Agudelo (1990) relata que a demanda mundial por sementes de Pinus maximinoi cresceu acentuadas, e que o Banco de Sementes da Escola Nacional de Ciências Florestais de Honduras teve dificuldades para abastecer os países compradores.

No Brasil, sua cultura ainda se encontra na fase experimental, demonstrando alto incremento volumétrico e poderá ser uma alternativa estratégica como fonte de madeira nas regiões tropicais e subtropicais. Essa espécie também poderá ser usada para produção de híbridos interespecíficos. O material genético disponível para plantio ainda demostra grande variação na forma e vigor, sendo assim a espécie ainda não foi adotada para produção em larga escala devido à falta de sementes com qualidade genética no mercado e informação sobre a qualidade da sua madeira. Porém, com trabalhos de seleção genética, a arquitetura da espécie em relação ao desenvolvimento pode ser melhorada. Alguns pesquisadores têm conduzido pesquisas com as espécies de pínus, como por exemplo, Florsheim (2010), Klock (2000), Moraes Neto et al. (2009 a,b), Moura et al. (2005), Poupin et al., 2005 e Wright et al. 1995). Diante deste contexto, o objetivo deste trabalho foi estimar variabilidade genética em testes de procedências e progênies em dois locais de plantio, bem como o ganho esperado com a seleção.

\section{MATERIAL E MÉTODOS}

Dois testes de procedências e progênies de Pinus maximinoi foram implantados em novembro de 1988 em duas áreas nas dependências da empresa Valor Florestal, Fazenda Moquém em Ventania-PR (Latitude: $24^{\circ} 07^{\prime}$, Longitude: $50^{\circ} 09^{\prime}$ ). O clima da região é subtropical úmido mesotérmico e a temperatura média é de $22^{\circ} \mathrm{C}$, e em anos com ocorrência de geadas frequentes e severas menor a $18^{\circ} \mathrm{C}$. A precipitação média anual é de aproximadamente $1.400 \mathrm{~mm}$, de acordo com a classificação 
climática de Köppen. Já o clima é predominantemente temperado úmido com verão quente e temperado úmido com verão temperado $(\mathrm{Cfa} / \mathrm{Cfb})$. Os solos encontrados são cambissolos, podzólios vermelho-amarelos, litólicos, latossolos vermelho-escuro e areias quartzosas (TRIANOSKI, 2012).

Os experimentos foram conduzidos sob o delineamento de blocos completos ao acaso, com nove blocos e seis plantas por parcela, em um espaçamento de 3 x 3 metros. As duas áreas foram compostas de progênies diferentes. Na Área 1 os tratamentos foram compostos por 63 progênies (Tabela 1) e na área 2 por 77 progênies de oito procedências (Tabela 2) de Honduras, México e Guatemala. As duas áreas não contêm as mesmas procedências.

Tabela 1. Locais de coleta de sementes e número de procedências e progênies de $P$. maximinoi da área 1.

Table 1. Seed collection sites and number of provenances and progenies of $P$. maximinoi from area 1.

\begin{tabular}{llll}
\hline Ordem & Municípios & No de Progênies & País \\
\hline 1 & La Canada & 7 & México \\
2 & Valle de Angeles & 9 & Honduras \\
3 & Marcala & 2 & Honduras \\
4 & Dulce N. de Copan & 14 & Honduras \\
5 & Coapilla & 6 & México \\
6 & San Jeronimo de Oaxaca & 7 & Guatemala \\
7 & San Juan Sacatepeques & 4 & Guatemala \\
8 & San Jeronimo & 13 & México \\
9 & Jaguariaíva (testemunha) & 1 & Brasil \\
\hline
\end{tabular}

Tabela 2. Locais de coleta de sementes e número de procedências e progênies de $P$. maximinoi da área 2.

Table 2. Seed collection sites and number of provenances and progenies of $P$. maximinoi from area 2.

\begin{tabular}{llll}
\hline Ordem & Municípios & No de Progênies & País \\
\hline 1 & Tapiquil & 16 & Honduras \\
2 & San Jeronimo & 14 & México \\
3 & Valle de Ageles & 12 & Honduras \\
4 & Coban & 5 & Guatemala \\
5 & Dulce N. de Copan & 15 & Honduras \\
6 & LA Canada & 2 & México \\
7 & Altamirando & 7 & México \\
8 & Tatumbla & 6 & Honduras \\
9 & Jaguariaíva (testemunha) & 1 & Brasil \\
\hline
\end{tabular}

A avaliação do diâmetro a altura do peito (DAP-cm) e a altura total (ALT-m) dos indivíduos foram conduzidas aos cinco anos de idade. Com base nesses dados foi estimado o volume cilíndrico $\left(\right.$ VOL-m $\left.3 / \operatorname{arv}^{-1}\right)$.

\section{Análise de deviance}

Foi realizada análise de deviance para determinar os efeitos de variação entre as procedências e entre e dentro das progênies para os caracteres de crescimento. Para isso foram realizadas duas análises: a primeira considerando todas as procedências na respectiva área, e a segunda em nível de progênies dentro de cada procedência.

\section{Análise entre procedência para cada local}

Os parâmetros genéticos e estatísticos foram estimados pelo método REML/BLUP (máxima verossimilhança restrita/ melhor predição linear não viciada), com o uso do software SELEGEN-REML/BLUP. O modelo usado na análise foi o de blocos completos casualizados, várias procedências, progênies de polinização aberta em um local:

$$
y=\mathbf{X} r+\mathbf{Z} a+\mathbf{W} p+\mathbf{T} s+e ;(\operatorname{RESENDE}, 2007) .
$$

em que; y é o vetor de dados, r é o vetor dos efeitos de repetição (assumidos como fixos) somados à média geral, a é o vetor dos efeitos genéticos aditivos individuais (assumidos como aleatórios), $\mathrm{p}$ é o vetor dos efeitos de parcela (assumidos como aleatórios), s é vetor dos efeitos de população ou procedência (aleatórios) e, e é o vetor de erros ou resíduos (aleatórios). As letras maiúsculas representam as matrizes de incidência para os referidos efeitos. 


\section{Análise individual}

Os componentes de variância e os parâmetros genéticos foram estimados com a utilização do método REML/BLUP (máxima verossimilhança restrita/melhor predição linear não viciada) (RESENDE, 2007). O modelo aplicado para análise de deviance dos testes de progênies foi o de blocos completo ao acaso, várias plantas por parcela, um só local. Para a análise dos dados assumiu-se que as progênies são de polinização aberta e a população encontra-se em conformidade com o equilíbrio de Hardy-Weinberg.

$$
y=\mathbf{X} r+\mathbf{Z} a+\mathbf{W} p+e ;(\text { RESENDE, 2007). }
$$

em que: y é o vetor de dados, r é o vetor dos efeitos de repetição (assumidos como fixos) somados à média geral, a é o vetor dos efeitos genéticos aditivos individuais (assumidos como aleatórios), p é o vetor dos efeitos de parcela, e é o vetor de erros ou resíduos (aleatórios). As letras maiúsculas representam as matrizes de incidência para os referidos efeitos.

\section{Estimativas de ganho esperado com a seleção}

Com o objetivo de selecionar indivíduos e progênies de procedências superiores em produção volumétrica para as gerações subsequentes de melhoramento, plantios comerciais e pomares clonais, os ganhos genéticos esperados com a seleção foram estimados a partir do ordenamento do valor genético aditivo individual, três intensidades de seleção: 2,5, 5 e 10\% (correspondendo 67, 135, 270 indivíduos na área 1 e 95, 190, 380 na área 2, respectivamente). As diferentes intensidades de seleção consideraram os resultados das análises genéticas estatísticas (BLUP). O caráter considerado na seleção foi o volume. O ganho genético em porcentagem (Gs\%) com a seleção correspondem a: Gs (\%) $=(\mathrm{Gs} / \hat{m}) \mathrm{x} 100$, em que $\hat{m}$ corresponde à média geral do experimento para determinado caráter.

\section{RESULTADOS E DISCUSSÃO}

\section{Análise entre Procedência}

A área 1 apresentou porcentagem de sobrevivência (82,04\%) superior a área 2 (78,59\%). Não houve diferenças na taxa de sobrevivência para as procedências devido ao local de plantio, confirmando a boa adaptação das procedências as condições edafoclimáticas consideradas.

Diferenças significativas $(\mathrm{p}<0,01)$ foram verificadas entre procedências, progênies e parcelas para os caracteres de crescimento na área 2. Para a área 1 foram observadas diferenças significativas $(\mathrm{p}<0,01)$ apenas entre progênies e parcelas para os todos os caracteres (Tabela 3). Esses resultados confirmam que a população de melhoramento de P. maximinoi introduzida no Brasil tem variação para caracteres de crescimento que podem ser explorados em ciclos subsequentes de melhoramento genético. Na Colômbia também verificaram variações significativas entre procedências e progênies de P. maximinoi para os mesmos caracteres avaliados no presente trabalho (WRIGHT et al., 1993). Outro estudo na Colômbia, Urrego e Lambeth (1988) relataram variações significativas entre procedências e entre progênies de P. maximinoi, para altura, DAP e volume. Esses resultados demonstram a existência de variações fenotípicas significativas em vários níveis, recomendando que diferentes métodos e intensidades de seleção podem ser aplicadas para o seu melhoramento genético, principalmente, as da área 2.

Tabela 3. Teste da razão de verossimilhança (LRT) entre procedências e progênies para os caracteres: altura total, diâmetro a altura do peito e volume em procedências de Pinus maximinoi aos cinco anos, Ventania, PR.

Table 3. Likelihood ratio test between provenances and progenies of the traits: total height, diameter at breast height and volume in provenances of Pinus maximinoi, five years old, in Ventania, PR.

\begin{tabular}{|c|c|c|c|c|c|c|}
\hline \multirow[b]{2}{*}{ Efeitos } & \multicolumn{3}{|c|}{ Área 1} & \multicolumn{3}{|c|}{ Área 2} \\
\hline & Altura (m) & DAP (cm) & Volume $\left(m^{3}\right.$.árv $\left.{ }^{-1}\right)$ & Altura (m) & DAP (cm) & Volume $\left(m^{3}\right.$.árv $\left.{ }^{-1}\right)$ \\
\hline Procedência & $4,03^{*}$ & $2,91 \mathrm{~ns}$ & $1,66 \mathrm{~ns}$ & $9,64^{* *}$ & $17,01^{* *}$ & $12,62^{* *}$ \\
\hline Progênie & $11,64^{* *}$ & $18,67^{* *}$ & $20,23^{* *}$ & $4,63^{* *}$ & $6,51^{* *}$ & $11,35^{* *}$ \\
\hline Parcela & $87,37^{* *}$ & $16,74^{\star *}$ & $24,58^{* *}$ & $151,02^{\star *}$ & $98,53^{\star *}$ & $91,44^{\star *}$ \\
\hline $\mathrm{CVe} \%$ & 6,66 & 12,37 & 25,93 & 7,44 & 13,72 & 27,63 \\
\hline$\hat{m}$ & 9,51 & 14,42 & 0,09 & 10,19 & 15,10 & 0,10 \\
\hline
\end{tabular}

LRT: Teste da razão de verossimilhança; $\chi^{2}$ qui-quadrado da deviance. *** e $^{*}$ LRT significativo $p<0,0$ I $(6,63)$, p $<0,05(3,84)$ e ns não significativo, $(\hat{m})$ média geral do experimento, (CVe\%) coeficiente de variação residual. 
As médias dos caracteres ALT, DAP e VOL na área 2 foram superiores a área 1, porém não muito discrepantes (Tabela 3). Na África do Sul, P. maximinoi apresentou crescimento de 5,4 a 5,7 m e 6,5 a 7,4 cm em ALT e DAP aos 4,8 anos, respectivamente (KIETZKA, 1987). Na Colômbia, o crescimento médio observado foi de 6,2 $\mathrm{m}$ e 8,8 cm em altura e DAP aos 2,9 anos, porém com alta incidência de fox-tail. Aos cinco anos de idade, as médias de crescimento de várias procedências implantadas na Colômbia, incluindo Altamirano, Cienega de Leon, Coapilla e Monte Cristo, foram de 10,4 m e 15,6 cm em altura e DAP (WRIGHT et al., 1993). No Brasil, um experimento instalado em Poços de Caldas (MG) composto por três espécies de Pinus mexicana revelou que P. tenuifolia (atual maximinoi) foi o que mais se destacou em altura e sobrevivência, atingindo uma altura média de 7,5 $\mathrm{m}$ aos 5 anos de idade (FERREIRA et al., 1972). O crescimento em ALT e DAP para as procedências e progênies em Ventania foram superiores quando comparado a outros testes com a mesma idade, principalmente, as da área 2.

A procedência La Canada, México apresentou a maior sobrevivência $(87,04 \%)$, enquanto que San Juan Sacatepeques, Guatemala a menor $(67,59 \%)$ na da área 1 . Na área 2, a maior sobrevivência foi observada para procedência Tapiquil, Honduras $(98,61 \%)$ e menor para Valle de Ageles, Honduras $(79,48 \%)$. As demais procedências da área 2 não diferiram muito com relação a sobrevivência. $\mathrm{Na}$ área 1 pode-se observar uma maior heterogeneidade na sobrevivência. O desempenho das testemunhas foi semelhante à média das progênies testadas $(97,45 \%)$. A sobrevivência para essa espécie tem sido relatada como variando de baixa a alta. A sobrevivência de $P$. maximinoi em Spitskop foi de 19\%. Por outro lado, a mesma espécie em Wilgeboom foi de $87 \%$. Nos ensaios com P. patula a espécie apresentou $89 \%$ de sobrevivência um pouco inferior a de P. elliottii $(97 \%) \mathrm{em}$ Spitskop e Wilgeboom (MITCHELL et al., 2012). Ettori et al., (2004), relataram taxas de sobrevivências das procedências Altamirano aos 10 anos (75,64\%), Copilla (75,81\%), Monte Cristo (70,37\%) e Cienega de Leon $(70,33 \%)$ foram menores as do presente estudo.

Os coeficientes de variação experimental ( $\mathrm{CVe}$ ) foram semelhantes nas duas áreas experimentais. Na área 1 observou-se o menor $C V e(6,66 \%)$ para o caráter altura, e na área 2 para o caráter volume $(27,63 \%)$. Os valores correspondem a uma alta precisão experimental, principalmente para os caracteres altura e DAP (Tabela 3). O CVe deve ser mantido em níveis adequados para não ocorrer viés nas estimativas dos parâmetros, e consequentemente na seleção de indivíduos e progênies. Resende (2002) relata que níveis satisfatórios desse parâmetro $C V e$ \% são menores que 10 . O volume sempre apresenta valores mais altos que os demais caracteres por ser obtido pela multiplicação dos dados de altura e diâmetro.

Tabela 4. Estimativa de parâmetros genéticos para os caracteres: altura total, diâmetro a altura do peito e volume em procedências de $P$. maximinoi aos cinco anos, Ventania, PR.

Table 4. Estimates of genetic parameters for the traits: total height, diameter at breast height and volume in provenances of $P$. maximinoi at five years, in Ventania, PR.

\begin{tabular}{|c|c|c|c|c|c|c|}
\hline & \multicolumn{3}{|c|}{ Área 1} & \multicolumn{3}{|c|}{ Área 2} \\
\hline Parâmetros & Altura (m) & DAP (cm) & Volume $\left(\mathrm{m}^{3} / \mathrm{arv}^{-1}\right)$ & Altura (m) & DAP (cm) & Volume $\left(\mathrm{m}^{3} / \mathrm{arv}^{-1}\right)$ \\
\hline$\hat{h_{a}^{2}}$ & $0,11( \pm 0,03)$ & $0,12( \pm 0,03)$ & $0,13( \pm 0,03)$ & $0,06( \pm 0,02)$ & $0,07( \pm 0,02)$ & $0,09( \pm 0,02)$ \\
\hline$\hat{C}_{c}^{2}$ & 0,12 & 0,05 & 0,06 & 0,15 & 0,12 & 0,11 \\
\hline$\hat{C}_{p}^{2}$ & 0,01 & 0,01 & 0,01 & 0,02 & 0,03 & 0,02 \\
\hline CVgi\% & 4,39 & 9,75 & 20,97 & 3,47 & 7,21 & 16,90 \\
\hline CVgp\% & 2,19 & 4,87 & 10,48 & 1,73 & 3,61 & 8,45 \\
\hline$\left(h_{a}^{2}\right)$ herdabilid & cias. (CV gi) & $\begin{array}{l}\qquad \hat{2}^{2} \\
\text { restrito; }\left(C_{C}\right) \\
\text { ente de variac }\end{array}$ & 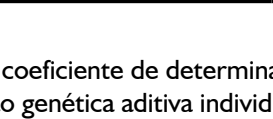 & & ela; $\left(\hat{C}_{p}^{2}\right)$ co & acão \\
\hline
\end{tabular}

O volume, caráter de maior interesse para o setor florestal, foi o que expressou o maior coeficiente de variação genética individual $\left(C V_{g i}\right)$ (Área $1=20,97 \%$ e Área 2=16,90\%) e coeficiente de variação genética entre progênies $\left(C V_{g p}\right)$ (Ârea $1=10,48 \%$ e Área $2=8,45 \%$ ), valores considerados de alta magnitude. Portanto, esse caráter demonstra ter maior potencial para a seleção (Tabela 4). $\mathrm{O}$ caráter altura apresentou o menor valor coeficiente de variação genética entre progênies $\left(C V_{g p}\right)$ 
(Área $2=1,73 \%$ ) esse valor significa que uma porcentagem pequena dos efeitos genéticos médios das progênies serão passados para próxima geração. Macedo et al., (2013) destaca que a caráter altura tem em geral menor variação genética entre progênies. Como esperado a variação individual é maior do que entre progênies, portanto a seleção individual deve ser priorizada. Os valores de variação genética individual e entre progênies são primordiais para que se encontrem indivíduos com potencial para compor os próximos ciclos subsequentes de melhoramento genético.

As estimativas de herdabilidade individual no sentido restrito foram baixas para os caracteres avaliados nas duas áreas (Tabela 4). A população da área 1 apresentou valores mais expressivos para esse parâmetro. Alguns métodos de seleção não devem ser adotados nessas áreas, como a seleção massal, que poderá resultar em baixos ganhos genéticos. Baixos coeficientes de herdabilidade, em nível de plantas individuais para caracteres de crescimento, têm sido encontrados em diversos trabalhos com espécies arbóreas (ETTORI et al., 2004; FREITAS et al., 2006; SATO et al., 2007; SEBBENN et al., 2009). Vários fatores podem afetar negativamente esse valor, inclusive uma amostragem não representativa da variabilidade genética das populações naturais da espécie.

\section{Análise individual}

Diferenças significativas entre e dentro de progênies para volume foram observadas apenas dentro das procedências de San Jerônimo de Baja Vera Paz e San Jerônimo de Oaxaca, e para Tatumbla e Valle de Ageles para área 1 e 2, respectivamente (Figura 1).
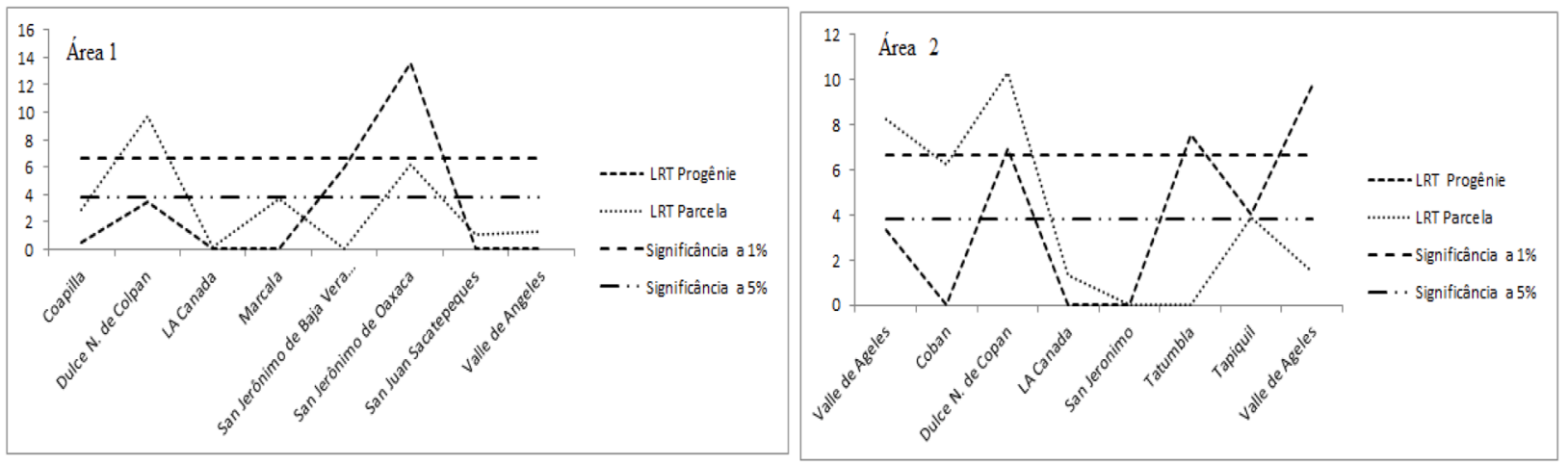

LRT: Teste da razão de verossimilhança; $\chi^{2}$ qui-quadrado da deviance. LRT significativo acima da linha pontilhada $p<0,05(3,84)$; acima da linha pontilhada $=p<0,0$ I $(6,63)$, e abaixo linha tracejada $=$ não significativo.

Figura 1. Teste da razão de verossimilhança para o caráter volume $\left(\mathrm{m}^{3}\right.$.arv $\left.{ }^{-1}\right)$ em progênies de $P$. maximinoi aos cinco anos para a área 1 , Ventania, PR.

Figure 1. Probability test for volume trait $\left(\mathrm{m}^{3}\right.$.tree $\left.{ }^{-1}\right)$ in $P$. maximinoi progenies at age five years for area 1 , in Ventania, PR.

As procedências Dulce N. de Copan, San Jeronimo de Oxaca, Tatumbla e Valle de Ageles foram as que mais contribuíram para as diferenças observadas na análise considerando o efeito de progênies dentro de cada procedência. $\mathrm{O}$ conhecimento da variabilidade genética é importante e necessário em populações de melhoramento, principalmente nas primeiras gerações. Assim, a infusão de novos genótipos em programas de melhoramento pode aumentar a recombinação e a variabilidade genética, possibilitando maiores ganhos nos ciclos subsequentes. Resende e Vencovsky (1990), descrevem que a melhor estratégia para a priorizar a conservação é aumentar o cruzamento entre indivíduos de diferentes populações e o aumento do tamanho efetivo de cada população.

As procedências de P. maximinoi mostraram bom desempenho em volume. Entre as várias procedências, as plantadas na área 2 foram as que apresentaram maior perspectiva de incremento volumétrico. Como a média de VOL foi maior na área 2, supõe uma diferenciação na variação ambiental ou superioridade das progênies desta área. Os efeitos ambientais mascaram o mérito genético dos indivíduos, assim quanto maior a proporção da variabilidade decorrente dos efeitos de ambiente em relação à variabilidade total, maior esforço deverá ser despendido na seleção dos genótipos superiores (BORÉM, 1998). Porém, no presente trabalho o delineamento ambiental teve um bom controle ambiental. Portanto, acredita-se que essa diferença deve-se ao mérito genético.

A herdabilidade estimada no sentido restrito $\left(\hat{h}_{a}^{2}\right)$ para todos os caracteres das procedências de ambas as áreas podem ser consideradas de média a alta magnitude conforme Resende (2002). As 
procedências La Canadá e San Jerônimo de Oaxaca na área 1 tiveram as maiores $\hat{h}_{a}^{2}(0,24$ e 0,4, respectivamente) (Figura 2).
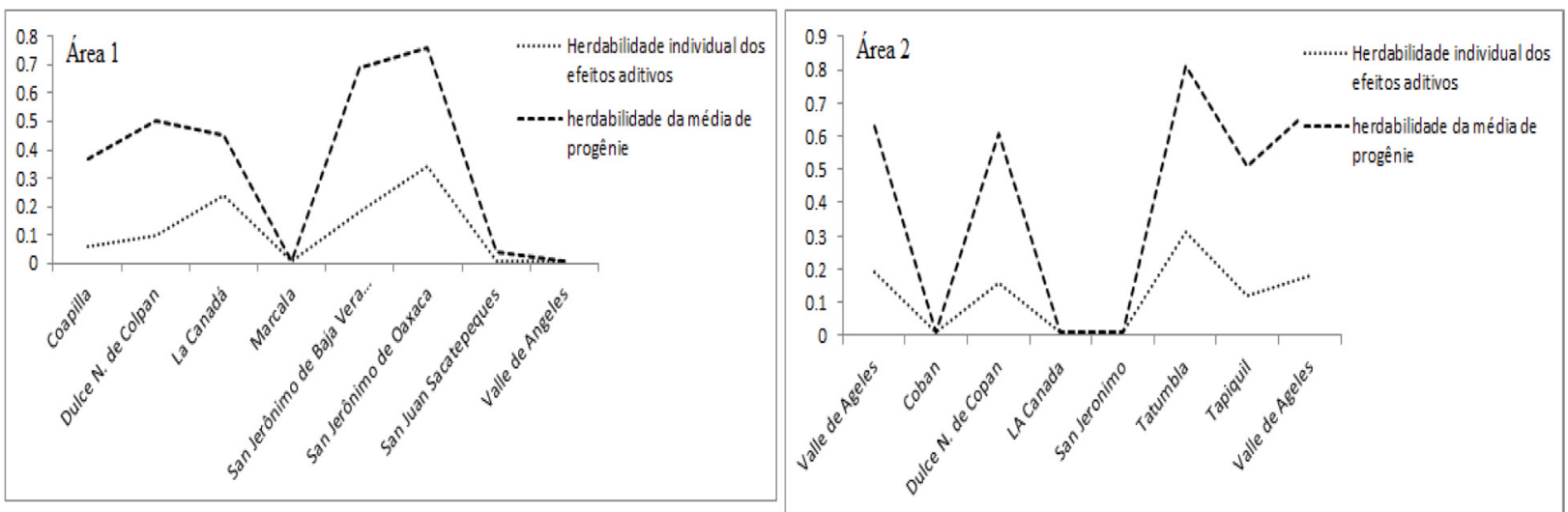

Figura 2. Estimativa de parâmetros genéticos para o caráter volume em progênies de $P$. maximinoi aos cinco anos para área 1 e 2, Ventania, PR.

Figure 2. Estimate of genetic parameters for volume in $P$. maximinoi progenies at five years for area 1 and 2 , in Ventania, PR.

$\mathrm{Na}$ área 2 as progênies Tatumbla e Valle de Ageles mostraram as maiores $\hat{h}_{a}^{2}(0,31$ e 0,18, respectivamente). Essas também foram as procedências com os maiores valores para herdabilidade media entre progênies $\left(\hat{h}_{m}^{2}\right)$. Isso indica que ganhos genéticos poderão ser obtidos com:aplicação de métodos de seleção entre progênies. A herdabilidade ao nível de média de progênies $\left({ }^{h}\right)$ indica que a seleção entre progênies poderá levar aos melhores progressos. A seleção de um maior número de indivíduos dessas procedências deve ser priorizada para compor os pomares de sementes por mudas e/ou a população de melhoramento.

O efeito de seleção de procedências considerando os componentes de média foi superior na área 2 (Tabela 5), enquanto que na área 1 não houve ganho em procedências. Maiores ganhos em nível de procedências serão obtidos com as procedências San Jeronimo, Coban, Altamirando, que pode ser explorada para o desenvolvimento de materiais genéticos superiores.

Tabela 5. Ganho esperado na seleção de procedências para caráter volume de $P$. maximinoi aos cinco anos, Ventania, PR. Table 5. Gain expected by selection of provenances for volume in $P$. maximinoi at age five, in Ventania, PR.

\begin{tabular}{|c|c|c|c|c|c|}
\hline \multicolumn{3}{|c|}{ Área 1} & \multicolumn{3}{|c|}{ Área 2} \\
\hline Procedências & Ĝs (\%) & Nova média $\left(\mathrm{m}^{3}\right.$.arv $\left.{ }^{-1}\right)$ & Procedências & Ĝs (\%) & Nova média $\left(m^{3} . a^{-1}\right)$ \\
\hline Dulce N. de Copan & 9,01 & 0,1027 & San Jeronimo & 4,62 & 0,1289 \\
\hline La Canada & 9,01 & 0,1027 & Coban & 3,41 & 0,1275 \\
\hline San Jeronimo & 9,01 & 0,1026 & LA Canada & 2,84 & 0,1268 \\
\hline Valle de Angeles & 9,01 & 0,1026 & Altamirando & 2,43 & 0,1262 \\
\hline San Jeronimo & 0,00 & 0,1026 & Dulce N. de Copan & 1,54 & 0,1252 \\
\hline Coapilla & 0,00 & 0,1026 & Tapiquil & 1,31 & 0,1242 \\
\hline Marcala & 0,00 & 0,1026 & Valle de Ageles & 0,00 & 0,1233 \\
\hline San Juan Sacatepeques & 0,00 & 0,1026 & Tatumbla & 0,00 & 0,1233 \\
\hline
\end{tabular}

Ĝs(\%):Ganho esperado na seleção

As estimativas de ganhos genéticos no ranking individual das progênies foram expressivas em todas as estimativas propostas. O ganho estimado para caráter VOL foi superior na área 2. Esses valores variaram de 9,04 a 13,0\% área 2 e de 5,88 a 9,77\% na área 1, conforme a pressão de seleção aplicada (Figura 3).

Esses resultados são expressivos para à formação de um pomar clonal ou produção de híbridos. Ganhos imediatos via seleção, depende da pressão de seleção, deixando a população mais homogênea e produtiva. Quando o objetivo de selecionar indivíduos para formação de pomar de semente (PSM) recomenda-se uma pressão de seleção mais branda. As amplitudes dos ganhos na seleção foram muito inferiores àquelas encontradas por Missio et al., (2004). Os autores observaram ganhos para volume de 10,04\% em progênies de P. caribaea var. bahamensis, com intensidade de seleção de 30\% 


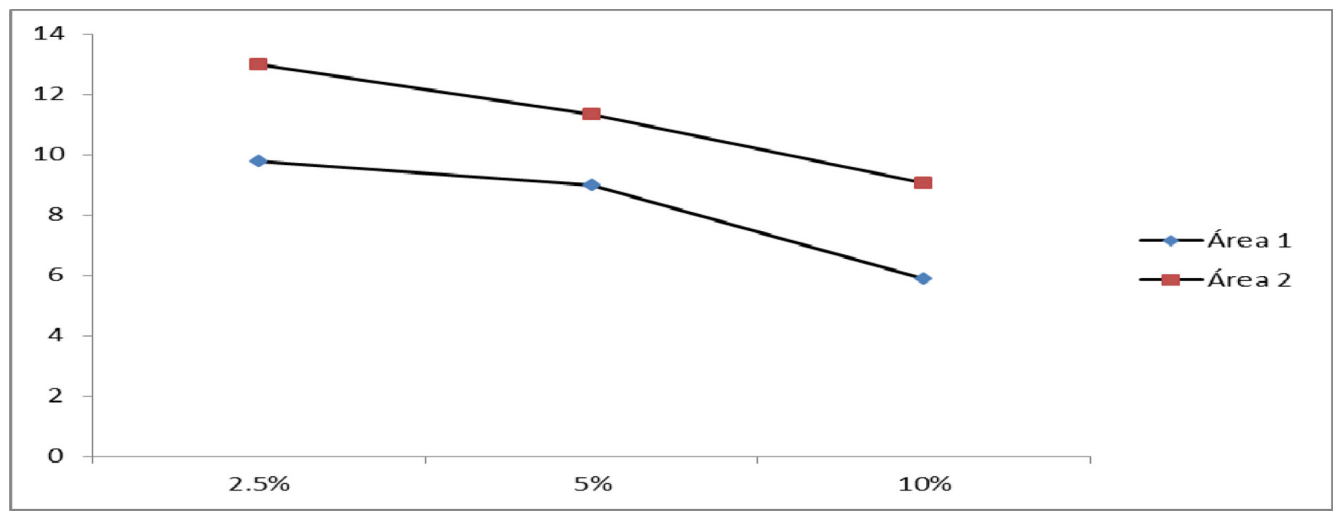

Figura 3. Estimativas de ganhos individual na seleção (GSI) com três intensidades (2,5, 5 e $10 \%$ para o caráter volume em progênies de $P$. maximinoi aos cinco anos para área 1 e 2 , Ventania, PR.

Figure 3. Estimate of individual gain in selection (GSI) with three intensities $(2.5,5$ and $10 \%)$ for volume trait in $P$. maximinoi progenies at five years for area 1 and 2, Ventania, PR.

Hodge e Dvorak (2012) relataram que o P. maximinoi apresentou melhor crescimento em volume $(42,2 \%)$ mesmo sem melhoramento genético do que $P$. patula já com algum grau de melhoramento genético. Conforme Nyoka et al. (2010), Hodge e Dvorak (2012) P. maximinoi tem um bom desempenho em crescimento e elevado potencial de adaptação se comparada às outras espécies de pínus ( $P$. patula, $P$. tedea, $P$. radiata), portanto pode ser considerado uma das principais espécies de pinheiro comerciais. Ganhos de 13 e 23\% a mais em volume foram obtidos para oito progênies de P. tecunumanii (NYOKA et al. 2010). Conclui-se que na região de Ventania-PR, P. maximinoi apresentou boa adaptabilidade e ótimo incremento, constituindo-se, portanto em uma espécie alternativa para diversificação dos plantios pínus na região.

\section{CONCLUSÕES}

Diferenças significativas entre as procedências e progênies de P. maximinoi apontam para possíveis ganhos genéticos significativos nas gerações subsequentes de seleção.

As procedências de Dulce N. de Colpan (Honduras) e San Jerônimo de Oaxaca (Guatemala) (área 1) e Tapiquil (Honduras) e Valle de Ageles (Honduras) (área 2) apresentam diferenças significativas dentro de procedências.

As procedências de $P$. maximinoi apresentam bom desempenho em crescimento na região de Ventania (PR), e deve ser considerada prioritária para plantios comerciais e estabelecimento de áreas de produção de sementes para essa espécie. Dentre as procedências testadas, San Jeronimo (México) teve as maiores taxas de incremento em volume.

A população de $P$. maximinoi da área 2 obtiveram ganhos com a seleção superiores a área 1, principalmente, quando usada uma pressão de seleção mais intensa.

\section{AGRADECIMENTOS}

Empresa Valor florestal, aos técnicos e funcionários da empresa Valor Florestal e Embrapa florestas pela coleta de dados e manutenção dos testes.

\section{REFERÊNCIAS BIBLIOGRÁFICAS}

AGUDELO C. N. J. Caracterización de Pinus caribaea Morelet, Pinus oocarpa Schiede y Pinus maximinoi H. E. Moore. Honduras: Escuela m Agrícola Panamericana, 1990. 51 p.

BORÉM, A. Melhoramento de plantas. 2.ed. Viçosa: Universidade Federal deViçosa, 1998. 453 p.

CESAR, E. R. G; SHIMIZU, J. Y; ROMANELLI, R. Variação entre procedências e progênies de Pinus oocarpa em Angatuba, SP. Boletim de Pesquisa Florestal, Colombo, n. 17, p.13-24, 1988. 
DVORAK, W. S; GUTIÉRRE Z, E.A.; GAPARE, W. J.; HODGE, G.R.; OSORIO, L.F.; BESTER,C.; KIKUTI, P. Pinus maximinoi. In: Conservation \& Testing of Tropical \& Subtropical Forest Tree Species. Raleigh: CAMCORE Cooperative, 2000. p. 107-127

ETTORI, L. C.; SATO, A. S.; SHIMIZU, J. Y. Variação genética em procedências e progênies mexicanas de Pinus maximinoi. Revista Instituto Florestal, São Paulo, v.16, n.1, p.1-9, 2004.

FERREIRA, M.; COUTO, H. T. Z.; MASCARENHAS SOBRINHO, J. Introdução de pinos mexicanos na região de Poços de Caldas. IPEF, Piracicaba, n. 4, p. 95-109, 1972.

FREITAS, M. L. M.; SEBBENN, A. M.; MORAES, E; ZANATTO, C. S.; VERARDI, C. K.; PINHEIRO, A. N. Parâmetros genéticos em progênies de polinização aberta de Cordia trichotoma (Vel.) ex. Steud. Revista Instituto Florestal, São Paulo, v. 18, p.95-103, 2006.

FLORSHEIM, S. M. B. Curvas de isopropriedades da madeira de árvores de Pinus caribaea var. bahamensis. 2010. 225 p. Tese (Doutorado em Ciências Florestais) - Escola Superior de Agricultura "Luiz de Queiróz", Universidade de São Paulo, Piracicaba, 2010.

HODGE, G. R.; DVORAK, W. S. Growth potential and genetic parameters of four Mesoamerican pines planted in the Southern Hemisphere. Southern Forests: a Journal of Forest Science, Pretoria, v. 74, n. 1, p. 27-49, 2012

KAGEYAMA, P. Y.; VENCOVSKY, R.; FERREIRA, M.; NICOLIELO, N. Variação genética entre procedências de Pinus oocarpa Schiede na região de Agudos - SP IPEF, Piracicaba, n.14, p.77-119, 1977

KIETZKA, J. E. Pinus maximinoi: a promising species in South Africa. South African Forestry Journal, Menlo Park, v. 145, n. 1, p. 33-38, 1987.

KLOCK, U. Qualidade da madeira de Pinus maximinoi H. E. Moore. 2000. 291 p. Tese (Doutorado em Ciências Florestais) - Universidade Federal do Paraná, Curitiba, 2000.

IBÁ - INDÚSTRIA BRASILEIRA DE ÁRVORES. Anuário estatístico, 2015 ano base 2014. São Paulo: IBA, 2015.

MACEDO, H. R.; FREITAS, M. L. M.; MORAES, M. L. T.; ZANATA, M.; SEBBENN, A. M. Variação, herdabilidade e ganhos genéticos em progênies de Eucalyptus tereticornis aos 25 anos de idade em Batatais-SP. Scientia Forestalis, Piracicaba, v. 41, p. 533-540, 2013

MALAN, F.S. The wood properties and sawn-board quality of. South African- grown Pinus maximinoi (HE Moore). Southern African Forestry Journal, Pretoria, n. 208, p. 39-47, 2006.

MISSIO, R. F.; CAMBUIM, J; MORAES, M. L. T.; PAULA, R. C. Seleção simultânea de caracteres em progênies de Pinuscaribaea Morelet var. bahamensis. Scientia Forestalis, Piracicaba, v. 66, p. 161 -168, 2004.

MITCHELL, R. G.; WINGFIELD, M. J.; HODGE, G. R.; DVORAK, W.; COUTINHO, T. A. Susceptibility of provenances andfamilies of Pinus maximinoi and Pinus tecunumanii to frost in South Africa, New Forests, Amsterdam, v. 44, n. 1, 2012.

MORAES NETO, S. P.; TELES, R. F.; RODRIGUES, T. O.; VALE, A. T.; SOUZA, M. R. Propriedades mecânicas de cinco procedências de Pinus caribaea var. hondurensis implantadas no cerrado do Distrito Federal. Boletim de Pesquisa e Desenvolvimento, Brasília, n. 251, 2009a, 20 p.

MORAES NETO, S. P.; TELES, R. F.; RODRIGUES, T. O. Caracterização da madeira de cinco procedências de Pinus tecunumanii implantadas no Cerrado do Distrito Federal. Boletim de Pesquisa e Desenvolvimento, Brasília, n. 233, 2009b, 30 p. 
Santos et al. - Identificação de Procedências e Progênies de Pinus maximinoi com potencial produtivo para madeira

MOURA, V. P. G.; VALE, A. T.; OLIVEIRA, I. R. M. Caracterização física e mecânica da madeira de Pinus tecunumanii com vistas à produção de móveis. Circular Técnica EMBRAPA/CENARGEN, Brasília, n. 43, p. 1-10, 2005.

NYOKA, B. I.;TONGOONA P.; GUMBIE C.M. Provenance productivity of high and low elevation Pinus tecunumanii in Zimbabwe. Silvae Genetica, Frankfurt, v. 59, p. 189-199, 2010

PRATA, J.G. Estudo da viabilidade tecnológica do uso de espécies de Pinus tropicais para produção de painéis colados lateralmente (Edge Glued Panels - EGP) 114 p. Tese (Doutorado em Ciências Florestais) Setor de Ciências Agrárias, Universidade Federal do Paraná, Curitiba, 2010.

PIRES, V. C. M.; MARTINS, K.; VILAS BOAS, O.; FREITAS, M. L. M. ; SEBBENN, A. M. . Variabilidade genética de caracteres silviculturais em progênies de polinização aberta de Pinus caribea var. bahamensis. Scientia Forestalis, Piracicaba, v. 41, n. 97, p. 113-119, 2013.

POUPIN, M.; ARCE-JOHNSON, P. Transgenic trees for a new era. In Vitro Cell Development Biology Plant, Heidelberg, v. 41, n. 2, p. 91-101, 2005.

RESENDE, M. D. V. Selegen-Reml/Blup: sistema estatístico e seleção genética computadorizada via modelos lineares mistos. Colombo: Embrapa Florestas, 2007. 359 p.

RESENDE, M. D. V. Genética biométrica e estatística no melhoramento de plantas perenes. Brasília: Embrapa Informação Tecnológica, 2002. 975 p. RESENDE, M. D. V.; VENCOVSKY, R. Condução e utilização de bancos de conservação genética de espécies de eucalipto. In: CONGRESSO FLORESTAL BRASILEIRO, 6., 1990, Campos do Jordão. Anais... São Paulo: Sociedade Brasileira de Silvicultura, 1990. p.434-439

SATO, A. S.; SEBBENN, A. M.; MORAES, E.; ZANATTO, A. C. S.; FREITAS, M. L. M. Seleção dentro de progênies de Eucalyptus resinifera aos 21 anos de idade em Luiz Antonio-SP. Revista Instituto Florestal, São Paulo, v.19, n. 2, p. 93-100, 2007

SEBBENN, A. M.; FREITAS, M. L. M.; ZANATTO, M. C. S.; MORAIS,E.; MORAES, M. A. Comportamento da variação genética entre e dentro de procedências e progênies de Gallesia integrifolia Vell. Moq. para caracteres quantitativos. Revista do Instituto Florestal, São Paulo, v. 21, n. 2, p.151-63, 2009.

SEBBENN, A. M.; SHIMIZU, J. Y., AGUIAR, A. V. Produção de resina de Pinus e melhoramento genético. In: SHIMIZU, J. Y. (Ed.). Pínus na silvicultura brasileira. Colombo: Embrapa Florestas, 2008. p. $193-206$

URREGO, J. B.; LAMBETH, C. C. Diferencias en cola de zorro entre familias y procedencias de Pinus maximinoi H. E. Moore en las montanas de Colombia. Investigación Forestal, Cali, n. 121, 4 p, 1988.

TRIANOSKI, R. Avaliação da qualidade da madeira de espécies de Pinus tropicais por meio de métodos convencionais e não destrutivos. 2012. 553 p. Tese (Doutorado em Engenharia Florestal) - Universidade Federal do Paraná, Curitiba, 2012.

WRIGHT, J.A.; WESSELS, A. Laboratory scale pulping of Pinus pseudostrobus, Pinus maximinoi e Pinus patula. IPEF International, Piracicaba, n. 2, p. 39-44, 1992.

WRIGHT, J. A.; SABOURIN, M. J.; DVORAK, W. S. Laboratory results of TMP and CTMP trials with Pinus patula, P. tecunumanii and P. caribaea var. hondurensis. Tappi Journal, Atlanta, v. 78, n. 1, p. 91-96, 1995.

WRIGHT, J. A. OSORIO, L. F.; LAMBETH, C. C. Development of a tree improvement program with Pinus maximinoi in Colombia. Forest Ecology and Management, Amsterdam, v. 62, p. 313-322, 1993.

Recebido em 05/04/2017

Aceito em 03/10/2017 